\title{
Thermal distribution in circular slabs: a thermographic method
}

\author{
by G. CESINI $\left({ }^{*}\right)$ M. PARONCINI $\left({ }^{*}\right)$ and R. RICCI $\left(^{*}\right)$
}

(*) Dipartimento di Energetica, Università degli Studi di Ancona, via Brecce Bianche, 60100 Ancona, Italy.

\begin{abstract}
Infrared thermography is commonly used as a qualitative method to investigate anomalies located inside solid bodies; the quantitative approach to the thermographic results is less extensive.

Nevertheless in many situations (e.g. industrial quality control or painting conditions) it is very important to find a thermographic method to obtain quantitative informations from the infrared pictures. The transient thermal behaviour of a circular slab with an internal air bubble is numerically and experimentally investigated. Two different materials have been used and the results are presented for different locations and sizes of the air bubble.
\end{abstract}

\section{Nomenclature}

$C_{p} \quad$ specific heat $(\mathrm{J} / \mathrm{Kg} \cdot \mathrm{K})$

$h \quad$ convective heat transfer coefficient $\left(\mathrm{W} / \mathrm{m}^{2} \cdot \mathrm{K}\right)$

$L \quad$ slab radius $(m)$

$L d \quad$ defect radius $(\mathrm{m})$

$L d^{*} \quad$ percentual defect radius, dimensionless $=100 \cdot L d / S$

$P d \quad$ defect depth (m)

${P d^{*}}^{*}$ percentual defect depth, dimensionless $=100 \cdot \mathrm{Pd} / \mathrm{S}$

$q \quad$ specific thermal flux $\left(W / \mathrm{m}^{2}\right)$

$R, Z \quad$ dimensional coordinates $(\mathrm{m})$

$R^{\prime}, Z^{\prime} \quad$ dimensionless coordinates

$S \quad$ slab thickness $(m)$

$S d \quad$ defect thickness (m)

$S d d^{*}$ percentual defect thickness, dimensionless $=100 \cdot S d / S$

$t$ time (s)

$t^{\prime} \quad$ Fourier number

$T$ temperature $\left({ }^{\circ} \mathrm{C}\right)$

$\mathrm{Te} \quad$ ambient temperature $\left({ }^{\circ} \mathrm{C}\right)$

Greek Symbols

$\alpha$

thermal diffusivity $\left(\mathrm{m}^{2} / \mathrm{s}\right)$

$\theta(R, Z, t)=\frac{T(R, Z, t)-T e}{\frac{q \cdot S}{\lambda}}$

$\lambda \quad$ thermal conductivity $(\mathrm{W} / \mathrm{m} \cdot \mathrm{K})$

$\rho \quad$ density $\left(\mathrm{Kg} / \mathrm{m}^{3}\right)$

\section{Introduction}

Infrared thermography is commonly used in various applications as a qualitative non destructive method of investigation; the quantitative inspection is less extensively used. 


\section{http://dx.doi.org/10.21611/qirt.1992.042}

In applications as the quality control of structures (avionic, civil and material fields) it is very important to find a procedure to investigate not only the presence but also the position and the dimensions of internal defects; therefore a quantitative analysis is needed.

In previous works the authors [1,2] studied the possibility of applications of infrared thermography to detect internal inhomogeneities in solid materials.

Combined with experimental investigations, a computer code was implemented which is based on the 3-D finite difference discretization method of unsteady thermal conduction problem in a parallelogrammic slab with an internal air bubble.

The comparison between numerical and experimental results was only qualitative and only for iron specimens. The thermal uneveness of the surface produced by a parallelogrammic air bubble located inside the specimen was evident for various positions and dimensions of the bubble.

Aim of the present work is to extend the previous results, by investigating how the thermal distribution on the surface of the slab depends on location and thickness of the air bubble and on the thermal conductivity.

\section{Numerical method}

In the present work the geometry of both the slab and the defect has axial symmetry, so that a 2-D finite difference scheme has been used to discretize the partial differential equation for unsteady heat conduction.

The geometry of the body sample is shown in figure 1 , together with the thermal boundary conditions. Due to the thermal symmetry of the system with respect to the central $Z$-axis the radial surface ( $R-Z$ plane) represents the heat transfer in the specimen.

The general equation of heat conduction in the slab is then:

$$
\rho C_{p} \cdot \frac{\partial T(R, Z, t)}{\partial t}=\lambda \cdot\left(\frac{\partial^{2} T(R, Z, t)}{\partial R^{2}}+\frac{\partial^{2} T(R, Z, t)}{\partial Z^{2}}\right)
$$

or in dimensionless form

$$
\frac{\partial \theta}{\partial t^{\prime}}=\frac{\partial^{2} \theta}{\partial R^{\prime 2}}+\frac{\partial^{2} \theta}{\partial Z^{2}}
$$

We have solved the equation (2) by means of two different finite difference methods:

a) A.D.I. - Alternating Direction Implicit method,

b) Explicit method,

using the same time step to compare the numerical results.

The time step used in the computations has been

$$
\Delta t^{\prime}=0.8 \cdot \Delta t_{\max }^{\prime}(\text { explicit method) }
$$

where $\Delta t^{\prime}$ max is obtained from the infinite-norm less than 1 condition (matrix method stability condition) applied to explicit algorithm.

In 2-D applications the A.D.I. method is unconditionally stable and more accurate than the explicit method. In fact the discretization errors $(O(\ldots))$ )are:

$$
\begin{array}{ll}
O\left(\Delta R^{2}, \Delta Z^{2}, \Delta t\right) & \text { for explicit algorithm } \\
O\left(\Delta R^{2}, \Delta Z^{2}, \Delta t^{2}\right) & \text { for A.D.I. algorithm }
\end{array}
$$




\section{http://dx.doi.org/10.21611/qirt.1992.042}

also the A.D.I. results have been more close to the experimental data.

To evaluate the thermal behaviour caused from the different position and thickness of the air bubble in the specimens, we assumed:

$$
\Delta \theta_{\max }=\left|\theta(0,1)-\theta\left(\frac{L}{S}, 1\right)\right|_{\max } \quad \text { and } \quad F o_{\max }=\frac{\alpha \cdot t_{\max }}{s^{2}}
$$

as reference parameters, where $t_{\max }$ is the time when $\Delta \theta=\Delta \theta_{\max }$ and $\Delta \theta_{\max }$ is the maximum module of $\Delta \theta$ in time.

To emphasize the sign of the thermal uneveness produced by the bubble air we have not used the module for $\Delta \theta_{\max }$ in the next figures.

\section{Experimental apparatus}

The heat source is an halogen light bulb voltage-controlled in order to provide different powers.

The lamp cone has the same diameter as the circular slab, the switching off time of the lamp is detected by means of digital hardware triggered from the switch lamp.

The thermographic investigations have been done with an AGA Thermovision 870 operating in the middle infrared band range (3-5 micron), the camera has a thermoelectric cooler of the infrared sensor whose sensitivity is about $0.2^{\circ} \mathrm{C}$.

The infrared images have been recorded on video-tape and successively analyzed by using an AGEMA digital image analysis system.

Many difficulties have been encountered to obtain an uniform heating of the slab. In fact small shifts of the lamp center produce high inhomogeneities in the thermal distributions in the slab.

At the moment we are realizing a thermal flowmeter plate to allow the adjustment of the bulb position and to evaluate the thermal heat flux released from the lamp with higher accuracy.

All specimens in the tests have been painted black with a special paint having emissivity 0.96 in the middle infrared range.

\section{Results}

Both numerical and experimental tests confirm the presence of a time where visibility has maximum.

Figure 2 shows numerical results obtained for a constant adimensional thickness $S d$ * of the inhomogeneity.

The results indicate that the position $P d^{*}$ of the inhomogeneity inside the slab can be univocally evaluated by knowing both $\Delta \theta_{\max }$ and $F o_{\max }$.

Furthermore, the different behaviour of $F_{O_{\max }}$ for the two investigated slab materials demonstrate that the Fourier number is not the dimensionless parameter suitable to generalize the thermal behaviour of our system.

Figure 3 shows the numerical results for both $P d^{*}$ and $S d^{*}$ constant values; the results are presented only for an aluminium slab.

From the analysis of the last figures, in connection with the results of figure 2 , it is possible to confirm the uniqueness of maximum visibility pair ( $\Delta \theta_{\max }, F_{o_{\text {max }}}$ ).

The experimental tests have been performed only for constant $S d$ * air bubble placed at three different positions inside aluminium and white cast iron specimens.

Figure 4 compare numerical and experimental results. 


\section{http://dx.doi.org/10.21611/qirt.1992.042}

The general behaviour of experimental tests matches with the numerical results. The quantitative comparison shows a difference between numerical and experimental results ranging from 10 to 30 percent .

This difference can be due to experimental errors connected with the technical characteristics of our thermocamera, with the thermal flux measurement, and with the influence of center lamp position on the thermal pattern of the surface.

\section{Conclusions}

The possibility of quantitative detection of internal anomalies in metallic slabs by thermographic methods has been investigated.

The technique is based on the radiative heating of one slab surface and on the simultaneous infrared observation on the opposite surface.

In these conditions it seems possible to obtain always only one pair ( $\Delta \theta_{\max }, F_{o_{\text {max }}}$ ) from which we can obtain the position and thickness of internal slab anomalies.

Experimental measurements confirmed the general thermal behaviour expected from the numerical methods.

Higher precision is required to the experimental apparatus in order to confirm the quantitative numerical results.

\section{REFERENCES}

1] CESINI (G.), GORI (F.), GUATTARI (G.) and LUCARINI (G.). - Thermal methods for painting diagnostics . I.I.C. VIIth Int. Congress, Oxford, England, 1973.

[2] CESINI (G.), PARONCINI (M.) and RICCI (R.). - A Thermographic method for nondestructive testing: numerical and experimental analysis. Fifth Conference on Thermogrammetry and Thermal Engineering, Budapest, 1987.

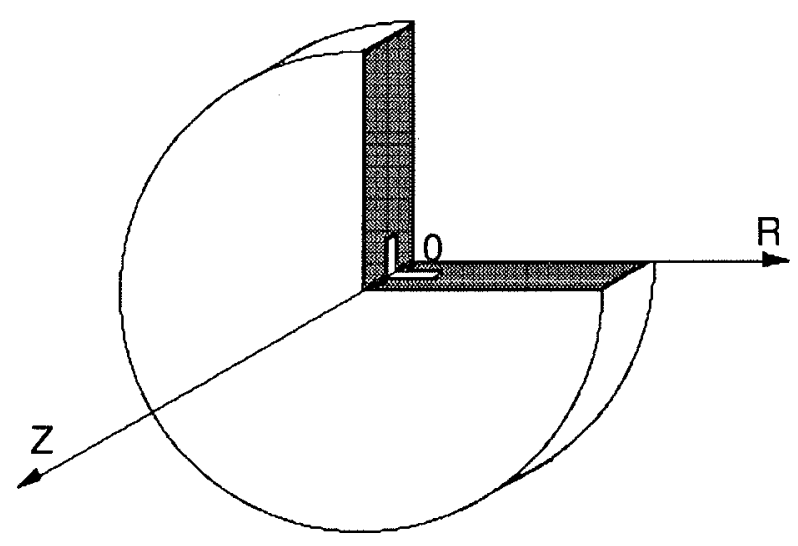

Fig. 1a. Circular slab with internal anomaly 


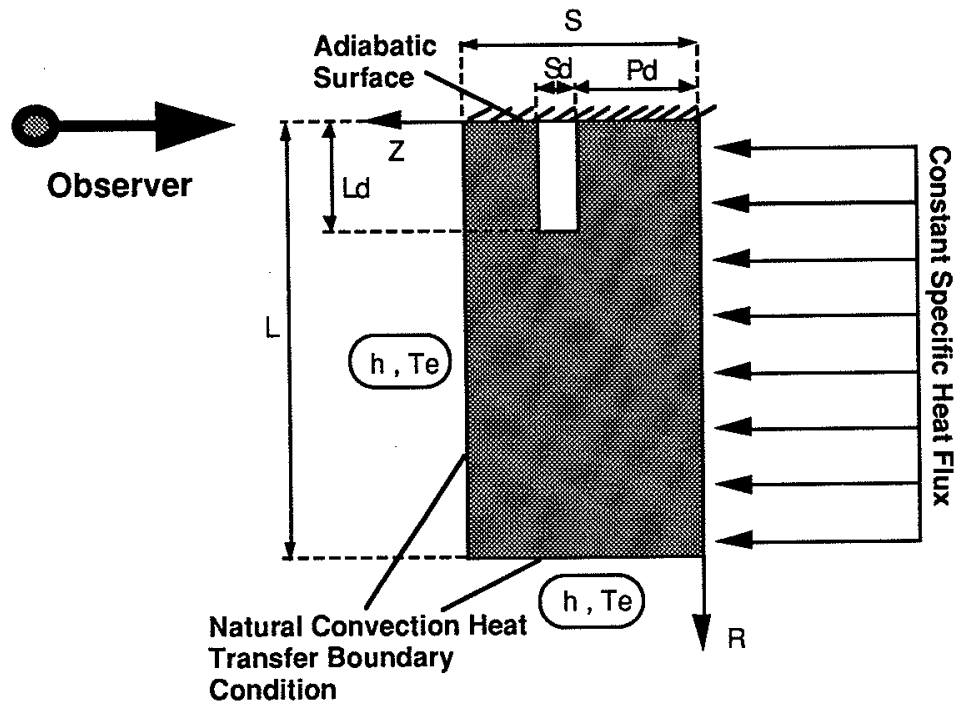

Fig. 1b. Two-dimensional domain used in numerical discretitation

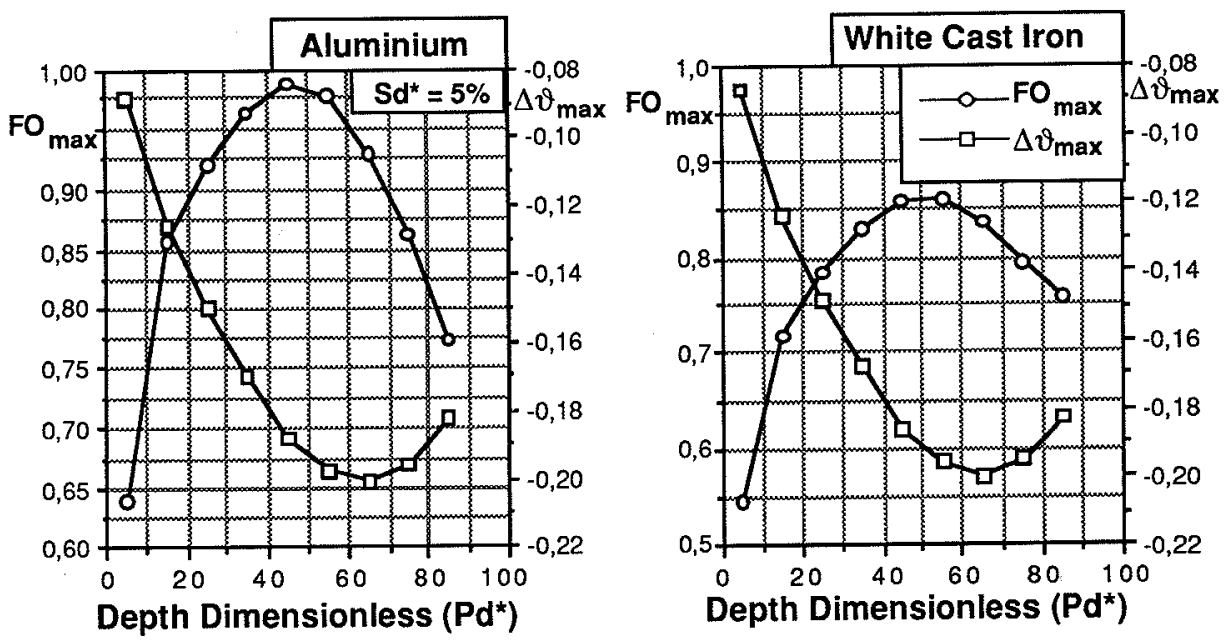

Fig .2. Numerical results for constant anomaly thickness 
http://dx.doi.org/10.21611/qirt.1992.042

$\Delta \vartheta_{\max }$

$-0,08$
$-0,10$
$-0,12$
$-0,14$
$-0,16$
$-0,18$
$-0,20$
$-0,22$

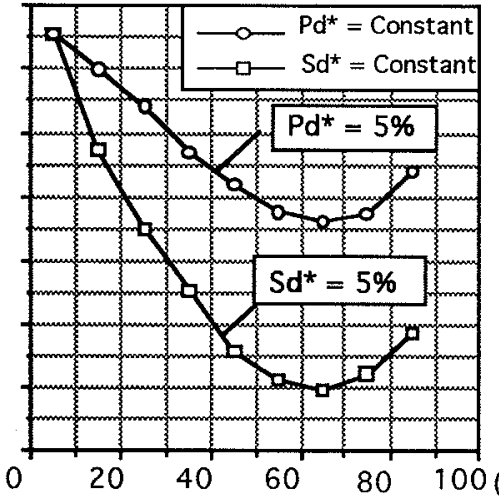

$\mathrm{FO}_{\text {max }}$

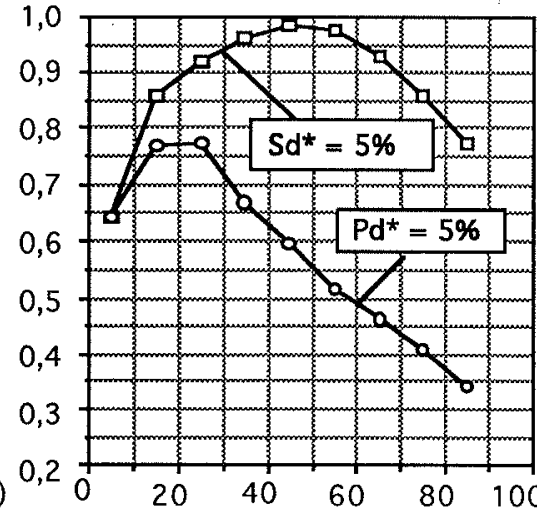

(\%)

Fig.3 . Numerical results for constant depth and for constant thickness
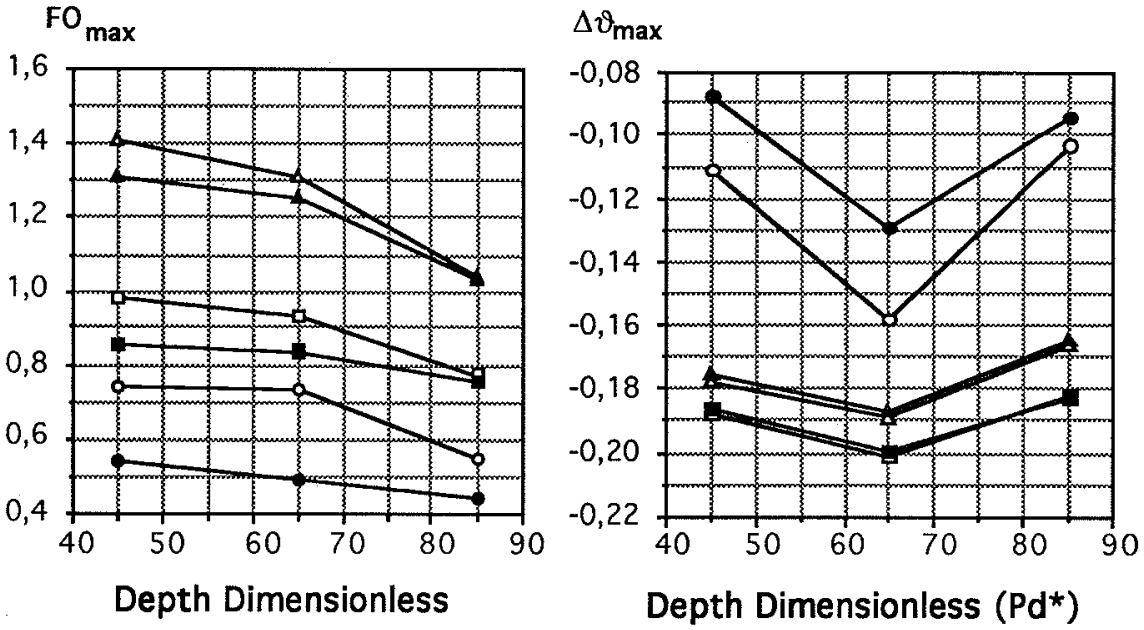

Fig. 4 . Comparisons between numerical and experimental results

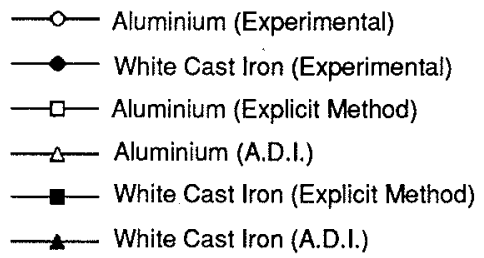

